Check for updates

Cite this: RSC Adv., 2017, 7, 54562

Received 31st October 2017

Accepted 22nd November 2017

DOI: 10.1039/c7ra11975e

rsc.li/rsc-advances

\section{Synthesis of an amphiphilic graft copolymer bearing a hydrophilic poly(acrylate acid) backbone for drug delivery of methotrexate $\uparrow$}

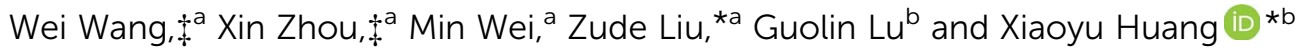

A well-defined amphiphilic graft copolymer consisting of a hydrophilic poly(acrylic acid) (PAA) backbone and hydrophobic poly(lactic acid) side chains was synthesized by the combination of reversible additionfragmentation chain transfer (RAFT) polymerization, ring open polymerization (ROP), and the graftingfrom strategy. RAFT homopolymerization of an $\mathrm{OH}$-containing tert-butyl 2-((4-hydroxy-butanoyloxy) methyl)acrylate (tBHBMA) monomer was first conducted to give a well-defined PtBHBMA homopolymer bearing pendant hydroxyls in every repeat unit. The pendant hydroxyls of PtBHBMA directly initiated the ROP of lactide without post-polymerization functionality transformation so as to provide a well-defined poly(tert-butyl acrylate)-g-poly(lactic acid) (PtBA-g-PLA) graft copolymer via the grafting-from strategy. The hydrophobic PtBA backbone was transformed into a hydrophilic PAA backbone to afford the target well-defined PAA-g-PLA amphiphilic graft copolymer $\left(M_{w} / M_{n}=1.17\right)$ containing equally-distributed carboxyls along the backbone. The PAA-g-PLA amphiphilic graft copolymer shows $\mathrm{pH}$-responsive micellization behavior and it can self-assemble into non-toxic large compound micelles under certain conditions for loading methotrexate (MTX). The in vitro accumulative release characteristics of MTXloaded micelles were investigated by UV/vis spectroscopy. In comparison with free MTX, the MTXloaded nanoparticles display higher cytotoxicity against MG-63 in 24, 48 and $72 \mathrm{~h}$.
\end{abstract}

\section{Introduction}

Biodegradable polymers have received increased attention and have been seen as a promising solution to the plastic waste issue because they can be broken down to environmentallyfriendly low molecular weight products such as water and carbon oxide. ${ }^{1,2}$ Among the many known biodegradable polymers, polylactides (PLAs) occupy a prominent position due to their biodegradability, biocompatibility, and ready availability from inexpensive renewable resources. ${ }^{3-8}$ PLAs can be fabricated into sutures, catheters, and prosthetic devices, and recently their application in tissue engineering has also been quite attractive. ${ }^{9}$ In addition, employing PLAs as carriers for drug delivery is now a hot research topic. ${ }^{\mathbf{1 0 - 1 2}}$ However, PLAs possess certain drawbacks including intrinsic hydrophobicity.

\footnotetext{
${ }^{a}$ Department of Orthopaedic Surgery, Renji Hospital, School of Medicine, Shanghai Jiaotong University, 1630 Dongfang Road, Shanghai 200127, People's Republic of China.E-mail: rjliuzd@126.com; Tel: +86-21-68383706

${ }^{b}$ Key Laboratory of Synthetic and Self-Assembly Chemistry for Organic Functional Molecules, Shanghai Institute of Organic Chemistry, Chinese Academy of Sciences, 345 Lingling Road, Shanghai 200032, People's Republic of China. E-mail: xyhuang@mail.sioc.ac.cn; Fax: +86-21-64166128; Tel: +86-21-54925310

$\uparrow$ Electronic supplementary information (ESI) available. See DOI: 10.1039/c7ra11975e

$\ddagger$ Both authors contributed equally to this work.
}

Therefore, PLAs are usually copolymerized to improve their physical, chemical, and mechanical properties. ${ }^{13,14}$

It is well known that the architecture of copolymers has great impact on their properties. ${ }^{15}$ Amphiphilic block copolymers with linear architecture are known to form different morphologies in specific solvents, which brings lots of potential applications including drug delivery, ${ }^{16-19}$ bioreactor, ${ }^{20,21}$ and catalysis. ${ }^{22,23}$ However, it has been showed that non-linear copolymers display fundamentally different properties compared to the linear copolymers. ${ }^{24,25}$ Amphiphilic graft copolymers, one of those polymers with non-linear structure, are endowed with some unusual properties such as exhibiting more stable aggregation in solution due to their confined and complicated structure. ${ }^{26}$ PLA-based amphiphilic graft copolymers in which PLA (or derivatives) is either backbone or side chain, could bring interesting properties such as increased hydrophilicity, ${ }^{27-30}$ higher degradability, ${ }^{28,29,31-33}$ and better histocompatibility. ${ }^{33,34}$ Although many works are reported on the synthesis of PLA-based amphiphilic block copolymers ${ }^{13}$ it is still unusual to see reports concerned with the synthesis of PLAbased graft copolymer because of synthetic obstacles including complicated topology and highly steric congestion. PLA could be used as the backbone of graft polymer, which could be realized by incorporating functionalities on PLA backbone. For instance, Riva et al. reported PLA-g-PEG amphiphilic graft copolymer by copolymerizing lactide with $\alpha$-chloro- $\varepsilon-$ 
caprolactone, through which the halogen was introduced into the backbone, followed by grafting PEG onto the backbone via click chemistry. ${ }^{35}$ However, this approach employing PLA as the backbone is not so convenient since that on one hand, it is not an easy task to incorporate functionalities such as halogen and hydroxyl into the backbone; on the other hand, because of the PLA-based backbone, many characteristics of PLA including crystallinity and mechanical properties might disappear. ${ }^{36,37}$ It is more common to introduce PLA as side chains while preparing PLA-based graft copolymers. ${ }^{38-41}$ But it is worthy noting that it is difficult to accomplish the synthesis of PLAbased amphiphilic graft polymers with a hydrophilic backbone whose every repeat unit is hydrophilic. Poly(2-hydroxyethyl methacrylate) (PHEMA), possessing a number of biomedical applications due to its biocompatibility and hydrophilicity, is often introduced as the backbone of PLA-based amphiphilic graft polymers. Atom transfer radical polymerization (ATRP) of HEMA is used to yield the backbones on which a portion of hydroxyls could initiate ring open polymerization (ROP) of lactide to give the desired amphiphilic graft copolymer.$^{42}$ Poly(vinyl alcohol) (PVA) is also often chosen as the backbone because of its intrinsic hydrophilicity and biogradability. Kissel et al. prepared PLA-based amphiphilic graft polymer using the pendant hydroxyls on PVA backbone to initiate ROP of lactide for affording PVA-g-PLA graft copolymer. ${ }^{43}$ In order to avoid to use stannous octoate as catalyst of ROP which would hinder the medical applications for the toxicity, Schue et al. synthesized PVA- $g$-PLA by employing $\mathrm{MgH}_{2}$ as catalyst of ROP of lactide initiated by PVA. ${ }^{44}$ Akashi $e t$ al. also accomplished the synthesis of PLA-based amphiphilic graft copolymers with poly $(\gamma$-glutamic acid) (PGA) containing carboxyls as hydrophilic backbone, through a coupling reaction between $\gamma$-PGA and PLA activated by 1-ethyl-3-(3-dimethylaminopropyl)carbodiimide..$^{45}$

It is clear that only some parts of the backbone of PLA-based graft copolymers are hydrophilic ${ }^{42-45}$ since a portion of hydrophilic functionalities including hydroxyls and carboxyls is converted to hydrophobic ester linkages for connecting PLA side chains. Meanwhile, the distribution of those residual hydrophilic groups along the backbone is also random, which means that the precise structure of amphiphilic graft polymers could not be guaranteed. To obtain the amphiphilic graft copolymers with precise structure, especially the hydrophilic functionalities of the backbone are equally distributed, the simplest way is to make every repeat unit of the backbone hydrophilic. To our best knowledge, none has ever reported the synthesis of PLA-based amphiphilic graft copolymers bearing a hydrophilic backbone whose every repeat unit is all hydrophilic for ensuring the equaled distribution of hydrophilic functionalities.

Herein, we report the synthesis of poly(acrylic acid)-g-poly(lactic acid) (PAA-g-PLA) amphiphilic graft copolymer as shown in Scheme 1, in which every repeat unit of hydrophilic backbone possesses a carboxyl and hydrophobic PLA side chains are connected to every repeat unit of backbone. In order to accomplish the synthesis of this new polymer, it is viable to use the monomer containing a ROP initiation group. As mentioned above, PLA side chains can be formed by ROP of lactide initiated by the pendant hydroxyls of the backbone. To achieve the construction of a fully hydrophilic backbone, PAA, a hydrophilic polymer with $\mathrm{pH}$ responsiveness, is chosen due to convenient and complete conversion from the hydrolysis of the corresponding polyacrylate. Based on these facts and previous work of our group on the synthesis of diverse amphiphilic graft polymers $^{46-50}$ and a new trifunctional inimer, ${ }^{51}$ tert-butyl 2-((4hydroxybutanoyloxy)methyl)acrylate ( $t$ BHBMA) which bears a polymerizable double bond, a hydroxyl as initiating group for ROP, and a tert-butoxycarbonyl as potential carboxyl, the welldefined PtBA-based backbone with pendant hydroxyl in every repeat unit is formed by RAFT homopolymerization of $t$ BHBMA followed by initiating ROP of lactide to form PLA side chains. The desired well-defined amphiphilic graft copolymer, PAA-gPLA, is finally achieved by selective acidic hydrolysis of P $t$ BAbased backbone using trifluoroacetic acid. PAA-g-PLA graft copolymer could self-assemble into large compound micelles in

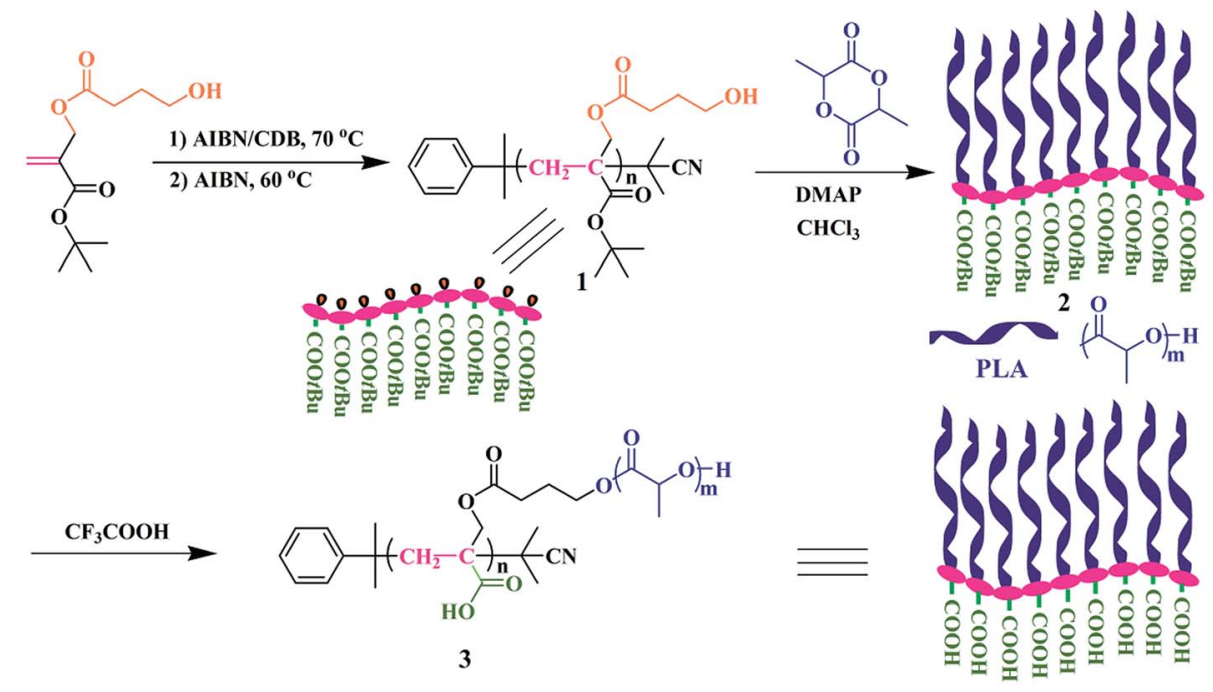

Scheme 1 Synthesis of PAA-g-PLA well-defined amphiphilic graft copolymer. 
aqueous media and is used to load methrotrexate (MTX). The morphology, size distribution, in vitro drug accumulative release, and cytotoxicity against MG-63 cells of the MTX-loading nanoparticles are investigated in details.

\section{Results and discussion}

\section{Synthesis of PtBA-g-PLA well-defined graft copolymer}

In 2013, our group developed a new trifunctional acrylate monomer of $t$ BHBMA, ${ }^{51}$ which consists of a polymerizable double bond, a hydroxyl as initiating group for ROP, and a tertbutoxycarbonyl as potential carboxyl. As it is well-known that hydroxyl survives the reversible-deactivation radical polymerization (RDRP) such as atom transfer radical polymerization (ATRP) and reversible addition-fragmentation chain transfer (RAFT) polymerization, therefore we can expect that the pendant hydroxyls of the homopolymer of $t$ BHBMA monomer can directly initiate ROP of lactone monomer including lactide to form the desired graft copolymer without polymeric functionality transformation, and tert-butoxycarbonyl can be readily converted to carboxyl for transforming hydrophobicity into hydrophilicity.

RAFT homopolymerization of $t$ BHBMA using AIBN as initiator and $\mathrm{CDB}$ as chain transfer agent was performed in DMF at $70{ }^{\circ} \mathrm{C}$ with a similar procedure ${ }^{51}$ to afford PtBHBMA 1 homopolymer bearing pendant hydroxyls in every repeat unit so as to achieve the synthesis of PLA-based amphiphilic graft copolymer with equally distributed carboxyls in the backbone. Fig. 1 shows GPC trace of PtBHBMA 1 homopolymer with a relative molecular weight $\left(M_{\mathrm{n}, \mathrm{GPC}}\right)$ of $4400 \mathrm{~g} \mathrm{~mol}^{-1}$ and a narrow molecular weight distribution $\left(M_{\mathrm{w}} / M_{\mathrm{n}}\right)$ of 1.26 . The chemical structure of P $t$ BHBMA 1 homopolymer is conformed by FT-IR (Fig. S1A $\dagger$ ) and ${ }^{1} \mathrm{H}$ NMR (Fig. 2A) and detailed discussion can be found in our previous report. ${ }^{51}$ The 'absolute' molecular weight of PtBHBMA 1 homopolymer $\left(M_{\mathrm{n}, \mathrm{NMR}}=6400 \mathrm{~g} \mathrm{~mol}^{-1}\right.$, DP of $\left.t \mathrm{BHBMA}=25.3\right)$ is determined from ${ }^{1} \mathrm{H}$ NMR of PtBHBMA 1 homopolymer using the same method described in our previous report. ${ }^{51}$ The minute amount of dithiobenzoate moiety was removed by AIBN according to a previous report ${ }^{52}$ for avoiding any possible effect of

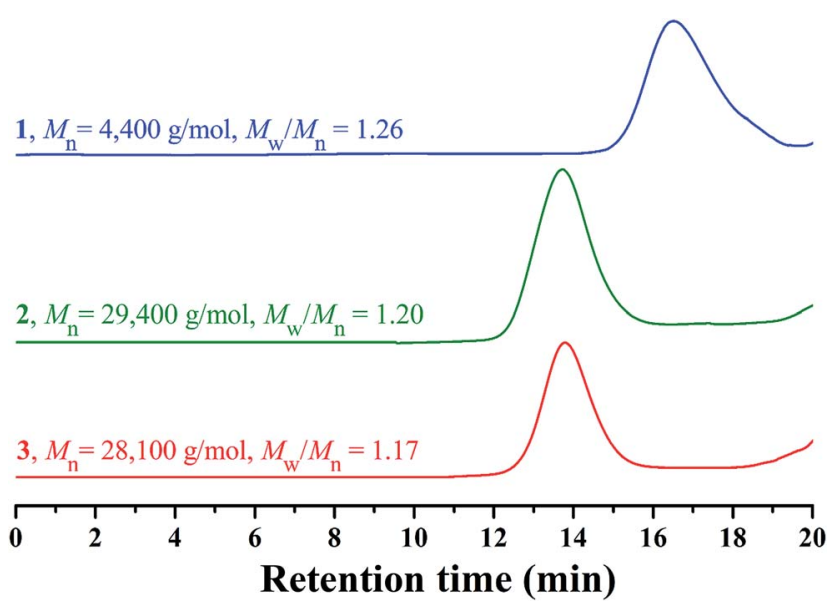

Fig. 1 GPC traces of PtBHBMA 1, PtBA-g-PLA 2 and PAA-g-PLA 3 in THF.

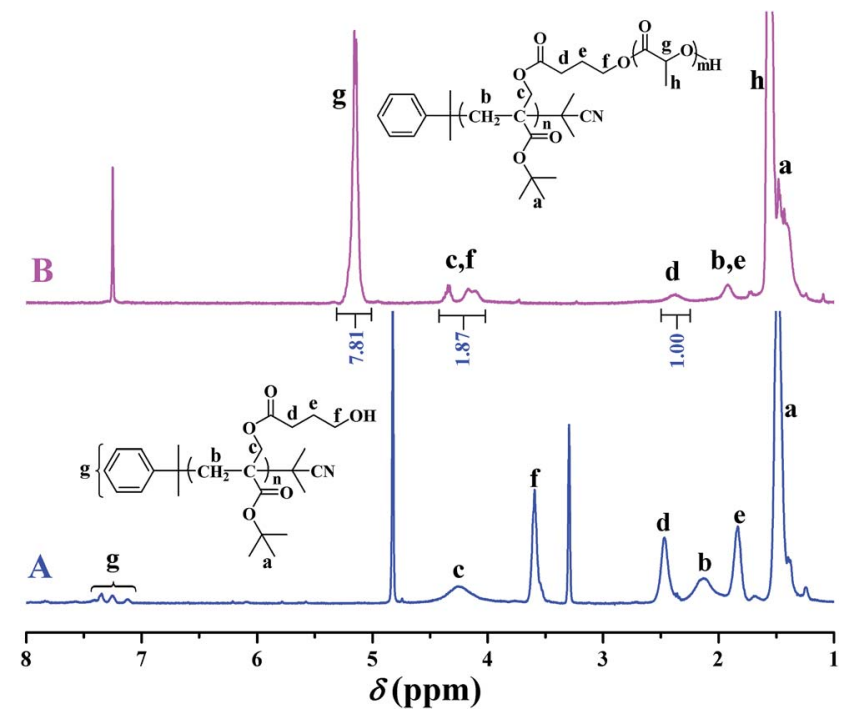

Fig. $2{ }^{1} \mathrm{H}$ NMR spectra of PtBHBMA 1 in $\mathrm{CD}_{3} \mathrm{OD}(\mathrm{A})$ and PtBA-g-PLA 2 in $\mathrm{CDCl}_{3}(\mathrm{~B})$.

dithiobenzoate end group on the following ROP of lactide and facilitating the observation of polymerization, which was evidenced by the color change from pink to white after the treatment with AIBN.

Recently, organocatalytic ROP of lactones including lactide has been developed very fast since this strategy could eliminate the usage of transition metal as catalyst which could prevent the application of corresponding polymers in biomedical and microelectronic fields. ${ }^{53,54}$ Therefore, the grafting-from strategy is employed for the construction of PtBA- $g$-PLA 2 graft copolymer, and ROP of lactide was initiated by the pendant hydroxyls of PtBHBMA 1 homopolymer using DMAP as organocatalyst in this report so as to attach PLA side chains onto every repeat unit of PtBA-based backbone. The purified product shows a unimodal and symmetrical elution peak (Fig. 1) with a narrow molecular weight distribution below 1.20 and its molecular weight $\left(M_{\mathrm{n}, \mathrm{GPC}}\right.$ $=29400 \mathrm{~g} \mathrm{~mol}^{-1}$ ) is much higher than that of the macroinitiator, which indicates the proceeding of ROP of lactide.

The chemical structure of PtBA- $g$-PLA 2 graft copolymer is characterized by FT-IR and ${ }^{1} \mathrm{H}$ NMR. The weak peak at $3510 \mathrm{~cm}^{-1}$ in FT-IR spectrum (Fig. S1A $\dagger$ ) is assigned to the terminal hydroxyl of PLA side chains while the signal originating from the pendant hydroxyls of PtBHBMA 1 homopolymer at $3435 \mathrm{~cm}^{-1}$ (Fig. S1B $\dagger$ ) is absent in Fig. S1A, $\dagger$ which implies all pendant hydroxyls are consumed to link PLA side chains. Moreover, the signal of carbonyl appears at $1756 \mathrm{~cm}^{-1}$ after ROP of lactide (Fig. S1A $\dagger$ ) in comparison with that located at $1740 \mathrm{~cm}^{-1}$ before ROP of lactide (Fig. S1B $\dagger$ ), this also witnessing the formation of PLA side chains. The peaks of both backbone and side chain are visible in ${ }^{1} \mathrm{H}$ NMR spectrum (Fig. 2B). Typical signals of PLA side chains appear at 1.56 (peak ' $h$ ') and 5.15 (peak ' $g$ ') ppm, which belong to three protons of $\mathrm{CHCH}_{3}$ and one proton of $\mathrm{CHCH}_{3}$, respectively. In particular, the signal originating from two protons of $-\mathrm{CH}_{2}$ group adjacent to pendant hydroxyls of PtBHBMA 1 homopolymer (peak ' $\mathrm{f}$ ' in 
Fig. 2A) disappears after ROP of lactide, which also clearly demonstrates that all pendant hydroxyls of P $t$ BHBMA 1 homopolymer initiated ROP of lactide, i.e. the number of grafted PLA side chain after ROP of lactide equals to that of $-\mathrm{CH}_{2} \mathrm{CH}_{2} \mathrm{CH}_{2}$ $\mathrm{OH}$ initiating group in $t$ BBPMA repeat unit before ROP of lactide, that is 25.3. The peaks at $16.7,69.1$, and $169.4 \mathrm{ppm}$ in ${ }^{13} \mathrm{C}$ NMR spectrum (Fig. S2 $\dagger$ ) could be assigned to carbons of methyl, methylidyne, and carbonyl in PLA side chains, respectively, which further illustrates the grafting of PLA side chains onto the backbone. This fact indicates that only the terminal hydroxyl of PLA side chains exists in the graft copolymer after ROP of lactide, which is consistent with the result of FT-IR. Thus, we can affirm the chemical structure of PtBA- $g$-PLA 2 graft copolymer.

Previous work showed that the molecular weight of graft copolymer determined by GPC might differ from the "real" value because of its branched structure. ${ }^{55}$ So, ${ }^{1} \mathrm{H}$ NMR was used to determine the 'absolute' molecular weight of PtBA- $g$-PLA 2 graft copolymer in the current case. The length of PLA side chain $\left(n_{\mathrm{LA}}\right)$ and the molecular weight of the graft copolymer $\left(M_{\mathrm{n}, \mathrm{NMR}}\right)$ were calculated according to eqn (1) $\left(S_{\mathrm{g}}\right.$ and $S_{\mathrm{d}}$ are the integration area of peak ' $\mathrm{g}$ ' at $5.15 \mathrm{ppm}$ (1 proton of $\mathrm{CHCH}_{3}$ ) of PLA side chain and peak 'd' at 2.37 ppm (2 proton of $\mathrm{CH}_{2} \mathrm{CH}_{2}$ $\mathrm{CH}_{2} \mathrm{O}$ ) of the backbone in Fig. 2B, respectively) and eqn (2) (25.3 is the number of $t \mathrm{BHBMA}$ repeated unit, 6400 and 72 are the molecular weights of PtBHBMA 1 macroinitiator and lactide monomer), respectively. The length of PLA side chain is estimated to be 15.6 and $M_{\mathrm{n}, \mathrm{NMR}}$ is evaluated to be $34800 \mathrm{~g} \mathrm{~mol}^{-1}$, obviously higher than that obtained from GPC.

$$
\begin{gathered}
n_{\mathrm{LA}}=2 S_{\mathrm{g}} / S_{\mathrm{d}} \\
M_{\mathrm{n}, \mathrm{NMR}}=6400+25.3 \times 72 \times n_{\mathrm{LA}}
\end{gathered}
$$

Thus, it can be concluded that a well-defined P $t$ BA- $g$-PLA 2 graft copolymer bearing a PtBA-based backbone (25.3 repeat units) and equally-distributed PLA side chains (15.6 repeat units per chain) connected to every repeat unit of the backbone has been successfully synthesized by ROP of lactide initiated by PtBHBMA 1.

\section{Conversion of PtBA- $g$-PLA to PAA-g-PLA}

It is well-known that hydrophobic PtBA can be easily transformed into hydrophilic PAA via acidic hydrolysis under mild conditions without affecting polyacrylate skeleton and other functionality. ${ }^{56,57}$ Therefore, PAA- $g$-PLA amphiphilic graft copolymer with a PAA backbone can be obtained from the precursor, $\mathrm{P} t \mathrm{BA}-\mathrm{g}$-PLA. Herein, $\mathrm{CF}_{3} \mathrm{COOH}$ was used to hydrolyze tert-butyoxycarbonyls of PtBA backbone in PtBA- $g$-PLA graft copolymer into carboxyls in $\mathrm{CH}_{2} \mathrm{Cl}_{2}$ at room temperature according to previous reports, ${ }^{\mathbf{5 6}, 57}$ in which excessive feeding of $\mathrm{CF}_{3} \mathrm{COOH}$ and long reaction time were employed to facilitate the hydrolysis of tert-butyoxycarbonyls. PLA side chains would keep inert during the hydrolysis process using $\mathrm{CF}_{3} \mathrm{COOH} .^{58}$

It can be seen from FT-IR spectra before (Fig. S1A $\dagger$ ) and after (Fig. S3 $\dagger$ ) the hydrolysis that a stronger peak at $3317 \mathrm{~cm}^{-1}$ attributed to the newly formed carboxyls appears in FT-IR spectrum after the hydrolysis in comparison with that before the hydrolysis. This fact apparently evidences the formation of PAA backbone. Fig. S3 $\uparrow$ also shows the remaining of typical signals of PLA (1760 and $1133 \mathrm{~cm}^{-1}$ ) segment in comparison with Fig. S1A, $\dagger$ which means that PLA segments indeed survive the hydrolysis. The hydrolyzed product also shows a unimodal and symmetrical elution peak (Fig. 1) with a similar molecular weight and molecular weight distribution compared to polymer 2, which affirms that polymeric skeleton was not affected during the hydrolysis. All these evidences confirm that PtBA- $g$-PLA 2 graft polymer has been selectively hydrolyzed in acidic environment for affording PAA- $g$-PLA 3 graft copolymer without affecting PLA side chains.

\section{pH-responsive micellization of PAA- $g$-PLA amphiphilic graft copolymer}

The amphiphilic nature of PAA- $g$-PLA 3 graft polymer, consisting of hydrophilic PAA backbone and hydrophobic PLA side chains, endows the polymer with capability to self-assemble in aqueous media. It has been reported that the solubility of PAA segment is related with the $\mathrm{pH}$ value of aqueous solution, i.e. PAA segment can become less soluble in acidic aqueous phase with a low $\mathrm{pH}$ due to the protonation of carboxyls in PAA segment. ${ }^{59}$ By lowering $\mathrm{pH}$ value of aqueous solution, PAA chain can turn from hydrophilic to hydrophobic and correspondingly, PAA- $g$-PLA 3 amphiphilic graft copolymer will also become completely hydrophobic. Therefore, fluorescence spectroscopy with $N$-phenyl-1-naphthylamine (PNA) as probe was employed to examine the pH-responsiveness of PAA- $g$-PLA 3 graft copolymer in aqueous media.

Fluorescence spectrum of PNA is sensitively affected by the environment and the polarity of its surrounding. ${ }^{60}$ In the presence of micelles, PNA is solubilized within the interior of the hydrophobic part showing high fluorescence intensity. Fig. 3 shows the relationship of fluorescence intensity of PNA emission band at $418 \mathrm{~nm}$ with $\mathrm{pH}$ of the aqueous solution of PAA-g-PLA 3 graft

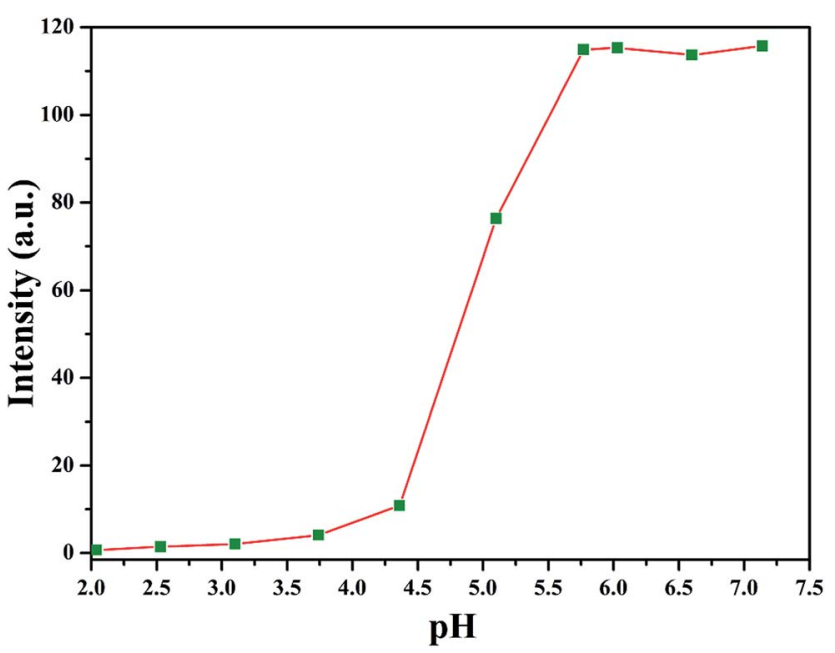

Fig. 3 Dependence of fluorescence intensity of PNA emission band at $418 \mathrm{~nm}$ on $\mathrm{pH}$ of the aqueous solution of PAA-g-PLA $3 \mathrm{graft}$ copolymer. 
copolymer and we can notice that the fluorescence intensity of PNA increases quickly when $\mathrm{pH}$ is above 4.36 because of the deprotonation of carboxyls to raise the hydrophilicity of the backbone for solubilizing PNA better, i.e. causing the rising of fluorescence intensity. When $\mathrm{pH}$ is above 5.77 , the fluorescence intensity of PNA reaches the highest value, suggesting the formation of compact and stable micelles. Fig. 4A shows that the average particle size of polymeric micelles formed by PAA- $g$-PLA 3 graft copolymer is about $144.7 \mathrm{~nm}$ obtained from DLS. Fig. 4B is TEM image of polymeric micelles, which exhibits the spherical micelles with a diameter of $c a$. 54-78 $\mathrm{nm}$. The smaller size observed by TEM compared to the result of DLS might be attributed to the shrinking of nanoparticles during the drying for the preparation of TEM specimen. Here, "spherical micelles" formed by PAA-g-PLA 3 amphiphilic graft copolymer should be large compound micelles, not common spherical micelles with only hydrophobic core covered by hydrophilic corona. That is, hydrophilic PAA segments form the corona of micelles and the core is consisted of numerous reverse micelles with islands of PAA segments in continuous phase of hydrophobic PLA segments. ${ }^{\mathbf{6 1 , 6 2}}$ All these facts demonstrate that the self-assembly of PAA-g-PLA 3 amphiphilic graft copolymer in aqueous media is $\mathrm{pH}$-dependent.

\section{Drug delivery of MTX using PAA-g-PLA graft polymer as carrier}

On the basis of aforementioned results, PAA- $g$-PLA 3 amphiphilic graft copolymer could assemble into large compound micelles with a diameter of about $144.7 \mathrm{~nm}$, which greatly benefits the application for drug delivery due to the so-called EPR effect. ${ }^{63,64}$ Therefore, we used PAA- $g$-PLA 3 amphiphilic graft copolymer as a carrier to prepare MTX-loading nanoparticles by dialysis approach $\left(M_{\mathrm{n}, \mathrm{cut}-\mathrm{off}}=3500 \mathrm{~g} \mathrm{~mol}^{-1}\right)$ against double-distilled water and the resulting solutions were then subjected to UV/vis spectroscopy for determining the drug loading content (DLC, 5.3\%) and drug loading efficiency (DLE, $20.6 \%$ ). The average particle size and size distribution of MTXloading micelles were determined by DLS and the size is about $189.3 \mathrm{~nm}$ (Fig. 4C), which is among the range of $50-200 \mathrm{~nm}$ suitable for EPR effect. TEM image reveals spherical shapes and a good dispersity with nanoscale sizes of about $83-98 \mathrm{~nm}$ (Fig. 4D).

In vitro release profiles of free MTX and MTX-loading micelles were assessed using the dialysis technique in PBS $(0.01 \mathrm{M}, \mathrm{pH}=5.0)$ at $37^{\circ} \mathrm{C}$. As shown in Fig. 5, free MTX almost completely released during $24 \mathrm{~h}$ while in the case of MTXloading micelles, about $33.1 \%$ of MTX released during the same time, and the accumulative release amount of MTX increases gradually with the extending of time, which indicates a significantly reduced burst release effect and an obvious sustained drug release behavior.

The in vitro cytotoxicity of free MTX, MTX-loading nanoparticles, and the bare PAA- $g$-PLA carrier were evaluated with MG-63 (human osteogenic sarcoma cell) using WST-8 based
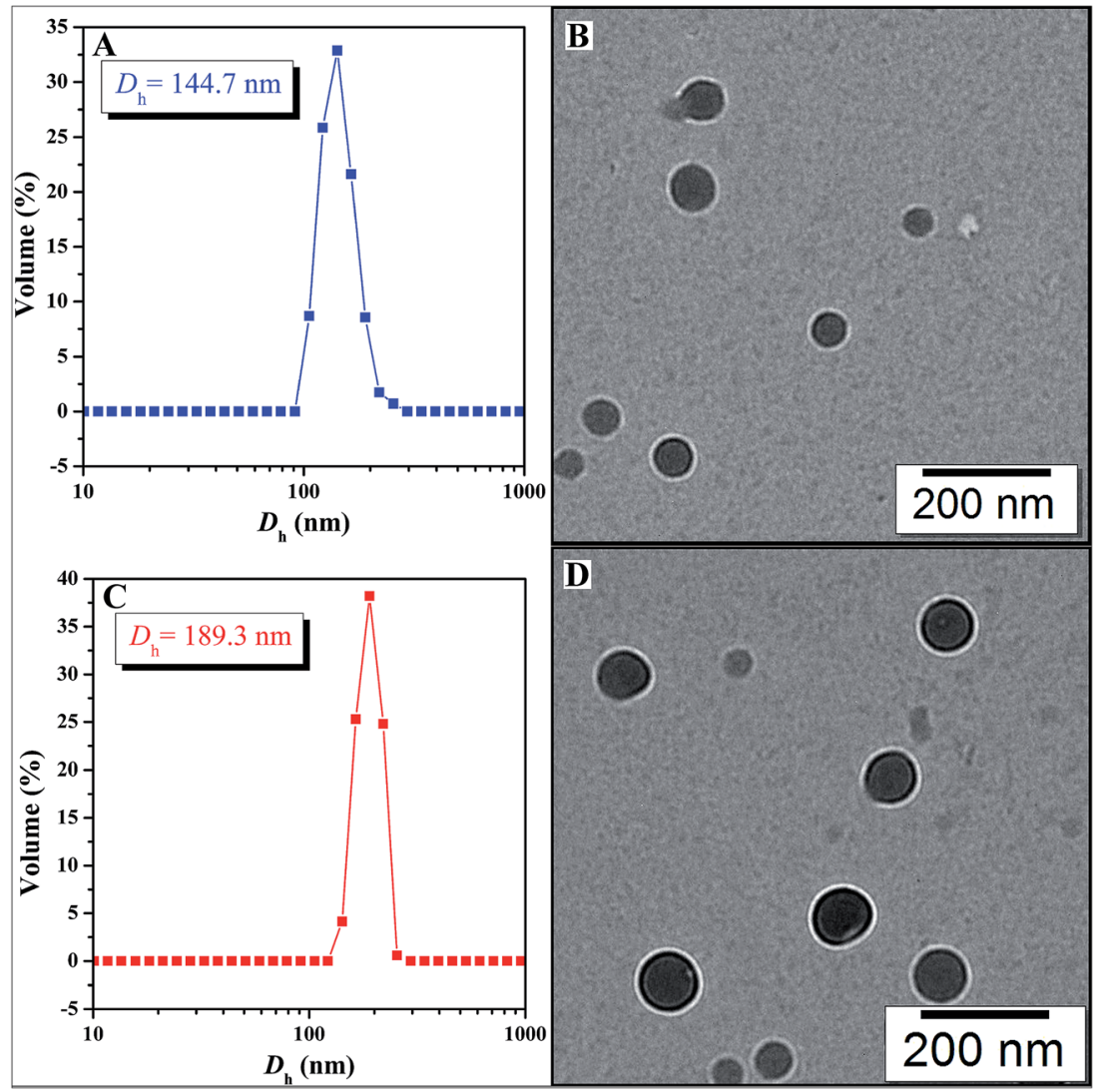

Fig. 4 Hydrodynamic diameter distributions of bare (A) and MTX-loading (C) micelles prepared from PAA-g-PLA 3; TEM images of bare (B) and MTX-loading (D) micelles prepared from PAA-g-PLA 3. 


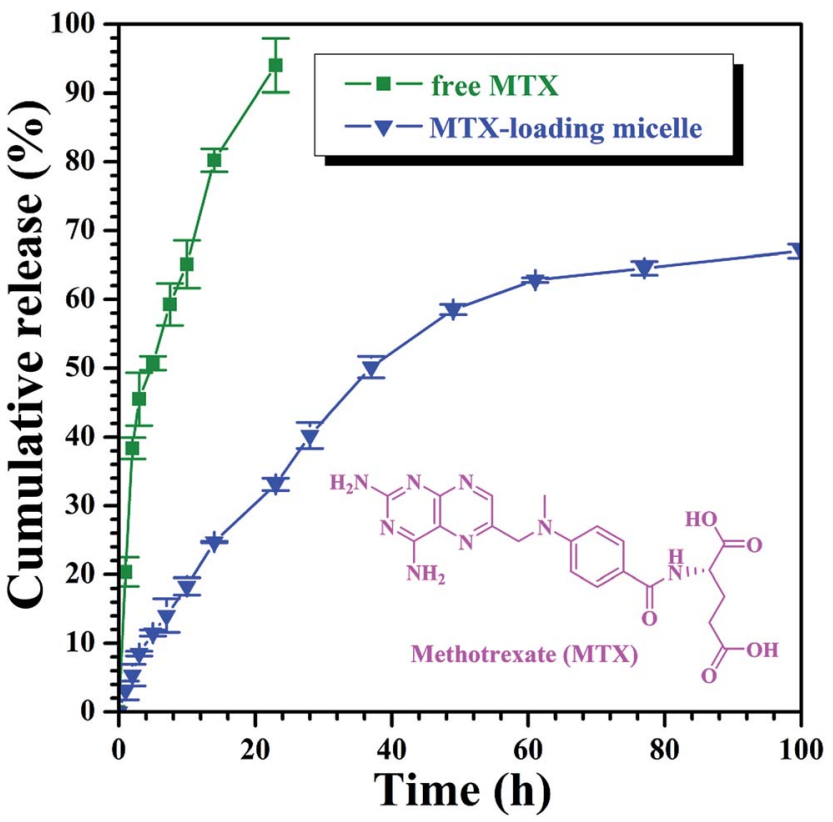

Fig. 5 In vitro MTX release profiles of free MTX and MTX-loading nanoparticles at $37^{\circ} \mathrm{C}$ under $\mathrm{pH}=5.0$.

colorimetric assay as shown in Fig. 6. High cell viability for MG$63(>93 \%)$ cells are found for various concentrations (up to 16 $\mu \mathrm{M})$ of PAA- $g$-PLA 3 micelles after incubating for $72 \mathrm{~h}$, this indicating the very low cytotoxicity of PAA-g-PLA 3 micelles and the suitability to be used as drug carrier. Both MTX-loading nanoparticles and free MTX are found to inhibit MG-63 cells and the relative cell viability declines monotonously with ascending the concentration of MTX. Obviously, MTX-loading nanoparticles show a higher cytotoxic effect on MG-63 cells in comparison with free MTX. For example, the viabilities of MG63 cells are $47.7 \%$ for free MTX and $36.4 \%$ for MTX-loading nanoparticles with a settled MTX concentration of $4 \mu \mathrm{M}$. Furthermore, it is worthy pointing out that MTX-loading nanoparticles with a low concentration of MTX exhibit a high cytotoxicity similar to free MTX with a high concentration. For instance, the viability of MG-63 cells is $40.6 \%$ for free MTX with a concentration of $8 \mu \mathrm{M}$, while it is $40.8 \%$ for MTX-loading nanoparticles with just $1 \mu \mathrm{M}$ of MTX, which clearly indicates that PAA-g-PLA could be used to load a low concentration of MTX for achieving the same treatment using a higher concentration of free MTX. Besides, the effect of incubation time on the cytotoxicity of MTX-loading nanoparticles and free MTX with a constant concentration of MTX $(16 \mu \mathrm{M})$ was investigated. It can be seen from Fig. 6B that MG-63 cells are inhibited by both free MTX and MTX-loading nanoparticles in a time-dependent mode. MTX-loading nanoparticles obviously exhibit a higher cytotoxicity effect on MG-63 cells compared to free MTX at every time interval. These above-mentioned results indicate that micelles prepared from PAA- $g$-PLA 3 amphiphilic graft copolymer could load MTX to improve the efficacy of MTX.

\section{Conclusions}

This report provides the synthesis and self-assembly studies on a new PLA-based amphiphilic graft copolymer, PAA-g-PLA. Target amphiphilic graft copolymer with equally distributed carboxyls in backbone was prepared by the acidolysis of hydrophobic backbone of a well-defined PtBA-g-PLA graft copolymer with a narrow molecular weight distribution $\left(M_{\mathrm{w}} / M_{\mathrm{n}}\right.$ $=1.20$ ), which was obtained by the combination of RAFT polymerization of $t$ BHBMA monomer and ROP of lactide, into hydrophilic PAA backbone. This amphiphilic graft copolymer is pH-responsive and can aggregate into large compound micelles in aqueous media. MTX was loaded by this kind of micelles selfassembled from PAA-g-PLA amphiphilic graft copolymer to provide MTX-loading nanoparticles with an average size of $c a$. $189.3 \mathrm{~nm}$ (DLS). It is illustrated that MTX-loading nanoparticles show sustained drug release profiles and inhibit MG-63 cells in a concentration- and time-dependent manner so as to exhibit a higher cytotoxicity effect compared to free MTX. All these
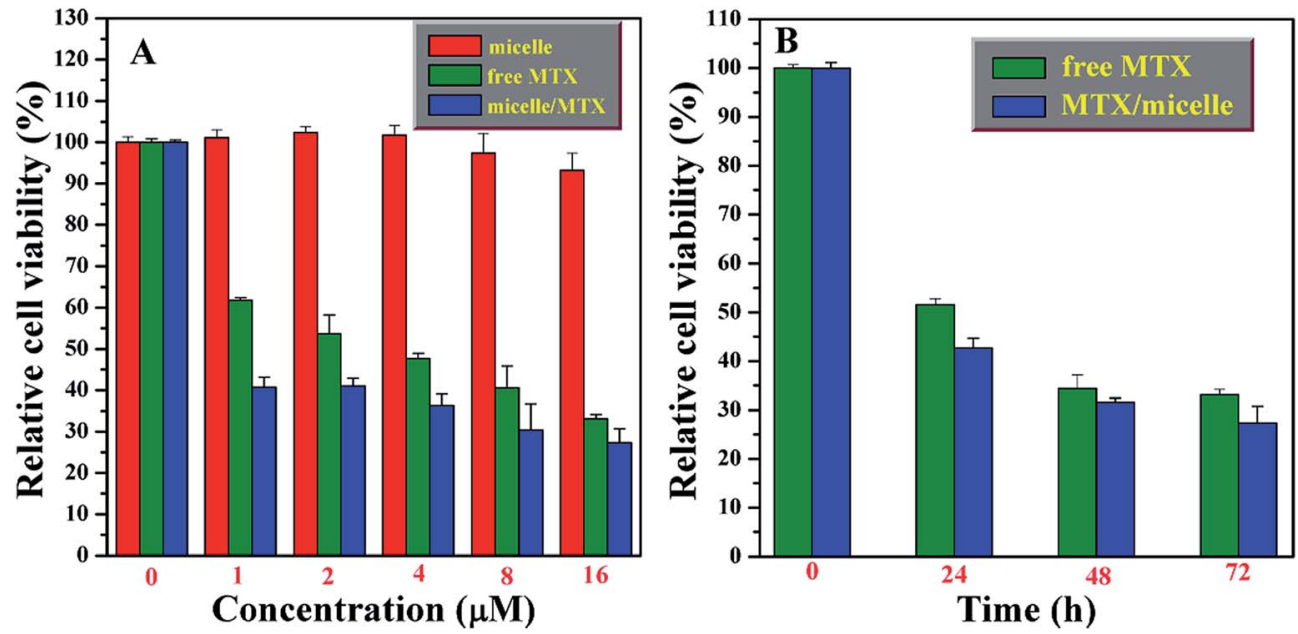

Fig. 6 Relative viabilities of MG- 63 cells after treatment with different concentrations of PAA-g-PLA 3 micelles, MTX-loading micelles and free MTX for $72 \mathrm{~h}$ (A) and with different time at a constant MTX concentration of $16 \mu \mathrm{M}(\mathrm{B})$. The experiments were repeated three times, all with similar results. The data are presented as the mean $\pm S$. D. (for each group, $n=3$ ). 
results suggest that PAA- $g$-PLA graft copolymer may be a good candidate as carrier for MTX or other anti-tumor drugs.

\section{Conflicts of interest}

There are no conflicts to declare.

\section{Acknowledgements}

The authors thank the financial support from National Natural Science Foundation of China (21474127), Strategic Priority Research Program of the Chinese Academy of Sciences (XDB20000000), and Shanghai Scientific and Technological Innovation Project (16JC1402500 and 16520710300).

\section{Notes and references}

1 L. S. Nair and C. T. Laurencin, Prog. Polym. Sci., 2007, 32, 762-798.

2 M. Akarami, I. Ghasemi, H. Azizi, M. Karrabi and M. Sayedabadi, Carbohydr. Polym., 2016, 144, 254-262.

3 G. Chen and M. K. Patel, Chem. Rev., 2012, 112, 2082-2099. 4 L. Yu, K. Dean and L. Li, Prog. Polym. Sci., 2006, 31, 576-602. 5 A. Raup, H. Wang, C. V. Synatschke, V. Jérôme, S. Agarwal, D. V. Pergushov, A. H. E. Müller and R. Freitag, Biomacromolecules, 2017, 18, 808-818.

6 M. Vert, Eur. Polym. J., 2015, 68, 516-525.

7 B. Gupta, N. Revagade and J. Hilborn, Prog. Polym. Sci., 2007, 32, 455-482.

8 Y. J. Xi, T. Song, S. Y. Tang, N. S. Wang and J. Z. Du, Biomacromolecules, 2016, 17, 3922-3930.

9 I. Manavitehrani, A. Fathi, Y. W. Wang, P. K. Maitz, F. Mirmohseni, T. L. Cheng, L. Peacock, D. G. Little, A. Schinder and F. Dehghani, Biomacromolecules, 2017, 18, 1736-1746.

10 V. Lassalle and M. L. Ferreira, Macromol. Biosci., 2007, 7, 767-780.

11 Y. Zhang, S. Liu, X. Wang, Z. Y. Zhang, X. B. Jing, P. Zhang and Z. G. Xie, Chin. J. Polym. Sci., 2014, 32, 1111-1118.

12 C. F. Yang, F. Sun, L. J. Zhang, G. D. Zhu, C. Y. Zhang and Y. Qian, Chin. J. Chem., 2012, 30, 1980-1986.

13 J. K. Oh, Soft Matter, 2011, 7, 5096-5108.

14 S. Corneillie and M. Smet, Polym. Chem., 2015, 6, 850-867.

15 X. M. Sun, J. X. Xu, J. B. Tang, M. H. Sui and Y. Q. Shen, Chin. J. Polym. Sci., 2011, 29, 427-430.

16 F. Ahmed and D. E. Discher, J. Controlled Release, 2004, 96, 37-53.

17 Y. Q. Zhu, B. Yang, S. Chen and J. Z. Du, Prog. Polym. Sci., 2017, 64, 1-22.

18 Y. F. Xiao, H. Sun and J. Z. Du, J. Am. Chem. Soc., 2017, 139, 7640-7647.

19 Q. M. Liu, L. W. Song, S. Chen, J. Y. Gao, P. Y. Zhao and J. Z. Du, Biomaterials, 2017, 114, 23-33.

20 P. Broz, S. Driamov, J. Ziegler, N. Ben-Haim, S. Marsch, W. Meier and P. Hunziker, Nano Lett., 2006, 6, 2349-2353.

21 C. Y. Yang, Q. Li, C. H. Cai and J. P. Lin, Langmuir, 2016, 32, 6917-6927.
22 S. B. Clendenning, S. Fournier-Bidoz, A. Pietrangelo, G. C. Yang, S. J. Han, P. M. Brodersen, C. M. Yip, Z. H. Lu, G. A. Ozin and T. Manners, J. Mater. Chem., 2004, 14, 1686-1690.

23 K. Nakajima, T. Fukui, H. Kato, M. Kitano, J. N. Kondo, S. Hayashi and M. Hara, Chem. Mater., 2010, 22, 3332-3339.

24 Y. L. Cai and S. P. Armes, Macromolecules, 2005, 38, 271-279.

25 Y. L. Cai, Y. Q. Tang and S. P. Armes, Macromolecules, 2004, 37, 9728-9737.

26 Y. Wang and S. M. Grayson, Adv. Drug Delivery Rev., 2012, 64, 852-865.

27 C. Nouvel, J. Raynaud, E. Marie, E. Dellacherie, J. L. Six and A. Durand, J. Colloid Interface Sci., 2009, 330, 337-343.

28 Y. Wan, X. Cao, Q. Wu, S. Zhang and S. Wang, Polym. Adv. Technol., 2008, 19, 114-123.

29 Y. Wan, X. Cao, S. Zhang, S. Wang and Q. Wu, Acta Biomater., 2008, 4, 876-886.

30 S. J. Carlotti, O. Giani-Beaune and F. Schue, J. Appl. Polym. Sci., 2001, 80, 142-147.

31 Y. Ohya, S. Maruhashi and T. Ouchi, Macromol. Chem. Phys., 1998, 199, 2017-2022.

32 Y. Ohya, S. Maruhashi and T. Ouchi, Macromolecules, 1998, 31, 4662-4665.

33 M. Li, Q. Huang and Y. Wu, Pest Manage. Sci., 2011, 67, 831836.

34 A. Lutzke, J. B. Tapia, M. J. Neufeld and M. M. Reynolds, ACS Appl. Mater. Interfaces, 2017, 9, 2104-2113.

35 R. Riva, S. Schmeits, F. Croisier, P. Lecomte and C. Jerome, Clin. Hemorheol. Microcirc., 2015, 60, 65-70.

36 C. H. Kim, K. Y. Cho and J. K. Park, Polym. Eng. Sci., 2001, 41, 542-553.

37 M. Labet and W. Thielemans, Polym. Chem., 2012, 3, 679684.

38 X. F. Niu, F. Tian, L. Z. Wang, X. M. Li, G. Zhou and Y. B. Fan, Chin. J. Polym. Sci., 2014, 32, 43-50.

39 J. Li, M. Kong, X. J. Cheng, Q. F. Dang, X. Zhou, Y. N. Wei and X. G. Chen, Int. J. Biol. Macromol., 2012, 51, 221-227.

40 T. Okuda, K. Ishimoto, H. Ohara and S. Kobayashi, Macromolecules, 2012, 45, 4166-4174.

41 H. Shinoda and K. Matyjaszewski, Macromolecules, 2001, 34, 6243-6248.

42 C. Zhao, D. Wu, N. A. Huang and H. Zhao, J. Polym. Sci., Part B: Polym. Phys., 2008, 46, 589-598.

43 A. Breitenbach and T. Kissel, Polymer, 1998, 39, 3261-3271. 44 N. Guerrouani, A. Mas and F. Schue, J. Appl. Polym. Sci., 2009, 113, 1188-1197.

45 Y. Zhu, T. Akagi and M. Akashi, Polym. J., 2013, 45, 560-566. 46 C. Feng, Y. J. Li, D. Yang, J. H. Hu, X. H. Zhang and X. Y. Huang, Chem. Soc. Rev., 2011, 40, 1282-1295.

47 S. J. Zhai, J. Shang, D. Yang, S. Y. Wang, J. H. Hu, G. L. Lu and X. Y. Huang, J. Polym. Sci., Part A: Polym. Chem., 2012, 50, 811-820.

48 S. J. Zhai, X. M. Song, C. Feng, X. Y. Jiang, Y. J. Li, G. L. Lu and X. Y. Huang, Polym. Chem., 2013, 4, 4134-4144.

49 X. Y. Jiang, X. Jiang, G. L. Lu, C. Feng and X. Y. Huang, Polym. Chem., 2014, 5, 4915-4925. 
50 G. L. Lu, H. Liu, H. F. Gao, C. Feng, Y. J. Li and X. Y. Huang, RSC Adv. , 2015, 5, 39668-39676.

51 X. M. Song, W. Q. Yao, G. L. Lu, Y. J. Li and X. Y. Huang, Polym. Chem., 2013, 4, 2864-2875.

52 S. Perrier, P. Takolpuckdee and C. A. Mars, Macromolecules, 2005, 38, 2033-2036.

53 N. E. Kamber, W. Jeong, R. M. Waymouth, R. C. Pratt, B. G. G. Lohmeijer and J. L. Hedrick, Chem. Rev., 2007, 107, 5813-5840.

54 S. A. Berg, H. Zuilhof and T. Wennekes, Macromolecules, 2016, 49, 2054-2062.

55 Z. F. Jia, Q. Fu and J. L. Huang, Macromolecules, 2006, 39, 5190-5193.

56 P. P. Li, Z. Y. Li and J. L. Huang, Macromolecules, 2007, 40, 491-498.
57 R. K. O'Reilly, M. J. Joralemon, K. L. Wooley and C. J. Hawker, Chem. Mater., 2005, 17, 5976-5988.

58 H. H. Liu, S. X. Li, M. J. Zhang, W. Shao and Y. L. Zhao, J. Polym. Sci., Part A: Polym. Chem., 2012, 50, 4705-4716.

59 S. C. Lee, K. J. Kim, Y. K. Jeong, J. H. Chang and J. Choi, Macromolecules, 2005, 38, 9291-9297.

60 L. C. You, F. Z. Lu, Z. C. Li, W. Zhang and F. M. Li, Macromolecules, 2003, 36, 1-4.

61 L. F. Zhang and A. Eisenberg, Science, 1995, 268, 1728-1731.

62 Y. S. Yu and A. Eisenberg, J. Am. Chem. Soc., 1997, 119, 83838384.

63 L. L. Li, F. Q. Tang, H. Y. Liu, N. J. Hao, D. Chen, X. Teng and J. Q. He, ACS Nano, 2010, 4, 6874-6882.

64 J. M. W. Chan, R. J. Wojtechki, H. Sardon, A. L. Z. Lee, C. E. Smith, A. Shkumatov, S. J. Gao, H. Kong, Y. Y. Yang and J. L. Hedrick, ACS Macro Lett., 2017, 6, 176-180. 